\title{
Air Entrainment by Contact Lines of a Solid Plate Plunged into a Viscous Fluid
}

\author{
Antonin Marchand, ${ }^{1}$ Tak Shing Chan, ${ }^{2}$ Jacco H. Snoeijer, ${ }^{2}$ and Bruno Andreotti ${ }^{1}$ \\ ${ }^{1}$ Physique et Mécanique des Milieux Hétérogènes, UMR 7636 ESPCI -CNRS, \\ Université Paris-Diderot, 10 rue Vauquelin, 75005, Paris \\ ${ }^{2}$ Physics of Fluids Group, Faculty of Science and Technology and MESA ${ }^{+}$Institute, \\ University of Twente, 7500AE Enschede, The Netherlands \\ (Received 13 September 2011; published 18 May 2012)
}

\begin{abstract}
The entrainment of air by advancing contact lines is studied by plunging a solid plate into a very viscous liquid. Above a threshold velocity, we observe the formation of an extended air film, typically 10 microns thick, which subsequently decays into air bubbles. Exploring a large range of viscous liquids, we find an unexpectedly weak dependence of entrainment speed on liquid viscosity, pointing towards a crucial role of the flow inside the air film. This induces a striking asymmetry between wetting and dewetting: while the breakup of the air film strongly resembles the dewetting of a liquid film, the wetting speeds are larger by orders of magnitude.
\end{abstract}

DOI: 10.1103/PhysRevLett.108.204501

PACS numbers: 47.20.Ma, 47.15.-x, 47.55.dd, 47.55.np

Objects that impact on a liquid interface can entrain small bubbles of air into the liquid. This happens, for example, when raindrops fall in the ocean [1] or when liquid is poured into a reservoir at sufficiently large speeds $[2,3]$. Such entrainment of air is often a limiting factor in industrial applications such as coating and nanoscale printing techniques, where the bubbles disturb the process $[4,5]$. A well studied case is the entrainment of air by very viscous jets impacting on a reservoir of the same liquid $[2,3,6,7]$. The onset of entrainment is essentially determined by the properties of the liquid, $U_{e} \sim \gamma / \eta_{\ell}$, which reflects a balance of the liquid viscosity $\eta_{\ell}$ and the surface tension $\gamma$. Changing the nature of the gas only has a minor influence on the entrainment process $[2,3]$.

A very different picture has emerged recently in the context of drops impacting on a wall, for which the presence of air has a dramatic effect [8]. It was found that splashing can be suppressed completely by reducing the air pressure to about a third of the atmospheric pressure. This caused huge excitement [9-13], in particular, because such a pressure reduction does not lead to any change of the gas viscosity $\eta_{g}$ : pressure only affects the gas density, and thus the speed of sound and the mean free path in the gas. A similar paradox is encountered for air entrainment by rapidly advancing contact lines, where a liquid advances over a surface that it partially wets [4,14-18]. Once again, it was found that depressurizing the gas leads to a significant increase of the threshold of air entrainment $[18,19]$. This contradicts the classical viewpoint that, for given wettability, the contact line speed depends mainly on the liquid properties as $\sim \gamma / \eta_{\ell}[14,20-22]$, with minor influence of the gaseous phase.

In this Letter we reveal the role of air for advancing contact lines in a paradigmatic system: a partially wetting solid plate is plunged into a reservoir of viscous liquid. The typical experimental scenario is presented in Fig. 1.
When the plate is plunged at small speeds, the contact line equilibrates to form a stationary meniscus and no air is entrained into the liquid [Fig. 1(a)]. Above a critical velocity, however, the contact line keeps moving downward into the reservoir and deposits a film of air [Figs. 1(b) and 1(c)], typically 10 microns thick. The substrate is sufficiently clean and the vibrations sufficiently low to avoid contact line pinning effects. The air film rapidly destabilizes after its formation, before ultimately decaying into small air bubbles (see Supplemental Material for experimental movies [23]). The liquid viscosity $\eta_{\ell}$ is varied over more than two decades by using silicon oils of different molecular weights. It is found that the entrainment speed $U_{e}$ changes much less than the expected scaling $\sim 1 / \eta_{\ell}$. Using an approximate hydrodynamic model we argue that this can be attributed to the flow of air into the strongly confined film, making the contact line velocity strongly dependent on both gas and liquid viscosities. This induces a striking asymmetry between wetting and dewetting: a liquid can advance much faster than it recedes, by orders of magnitude.

We explore how this process of air entrainment is influenced when varying the liquid viscosity. We therefore use silicon oils (PDMS, Rhodorsil 47V series) with dynamic viscosities $\eta_{\ell}=0.02,0.10,0.5,1.0$ and $5 \mathrm{~Pa} \cdot \mathrm{s}$. These liquids are essentially nonvolatile, insensitive to contamination, while the surface tension $\gamma=22 \mathrm{mN} \cdot \mathrm{m}^{-1}$ and density $\rho=980 \mathrm{~kg} \cdot \mathrm{m}^{-3}$ are approximately constant for all viscosities. The reservoir containing the liquid is a transparent acrylic container of size $29 \times 15 \times 13.5 \mathrm{~cm}$, which is much larger than the capillary length $\ell_{\gamma}=$ $(\gamma / \rho g)^{1 / 2}=1.5 \mathrm{~mm}$. The substrate consists of a silicon wafer (circular, diameter $10 \mathrm{~cm}$ ), which is coated by a thin layer of fluorinated material [FC 725 (3M) in ethyl acetate]. For all liquids, this results in static contact angles between $51^{\circ}$ and $57^{\circ}$. The wafer is clamped onto a $10 \mathrm{~mm}$ 


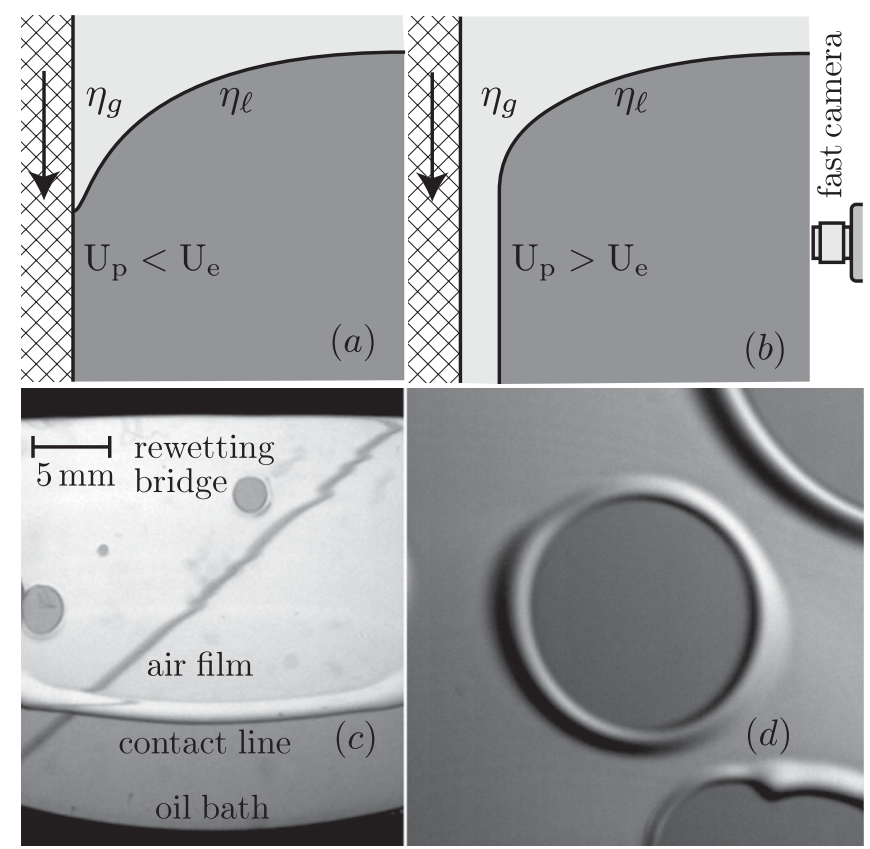

FIG. 1. Air entrainment by a contact line when a solid plate is plunged into a viscous liquid. (a) Sketch of dynamical meniscus below the threshold of air entrainment, and (b) above the threshold of air entrainment, where a film of thickness $h$ develops. A highspeed camera is placed perpendicularly to the substrate. (c), (d) Front views of the air film entrained into a viscous silicone oil $\left(\eta_{\ell}=0.1 \mathrm{~Pa} \cdot \mathrm{s}\right)$. (c) The extended air film is formed behind the contact line and moves downwards into the bath (taken $70 \mathrm{~ms}$ after plunging). The film is destroyed by the formation of "rewetting" bridges. The diagonal dark line is the reflection of a straight wire that gives an impression of the interface profile. (d) Zoom of the rewetting bridge, which is the inverse of the dewetting of a liquid film. Once the oil reestablishes contact with the solid, a growing circular zone invades the air film.

thick metallic blade screwed to a $50 \mathrm{~cm}$ long high-speed linear stage. The combination of controlled speeds and very viscous liquids avoids complexities of splashing as well as the formation of interface cusps [4,14,16-18]. In addition the effect of inertia is eliminated both in the gas and in the liquid: the Reynolds number based on the film thickness $h$ and on the entrainment threshold $U_{e}$ is at worst $\sim 0.2$, but typically orders of magnitude smaller. For each liquid, we plunge the wafer into the reservoir at different plate velocities $U_{p}$, up to $0.7 \mathrm{~m} / \mathrm{s}$. The process is recorded using a high-speed Photron SA 3 camera $(2000 \mathrm{~Hz}, 1024 \times$ 1024 pixels). The contact line velocities are extracted from space-time diagrams using a correlation technique with a subpixel resolution, leading to a precision of within a percent. Reproducibility is within $15 \%$. The film thickness $h$ is determined by dividing the volume of air entrained in the bath by the surface of the film (see experimental methods in the Supplemental Material [23]). The resulting thickness is reported in Fig. 2(c) for a fixed plate velocity, and is of the order of $10 \mu \mathrm{m}$ for all viscosities.
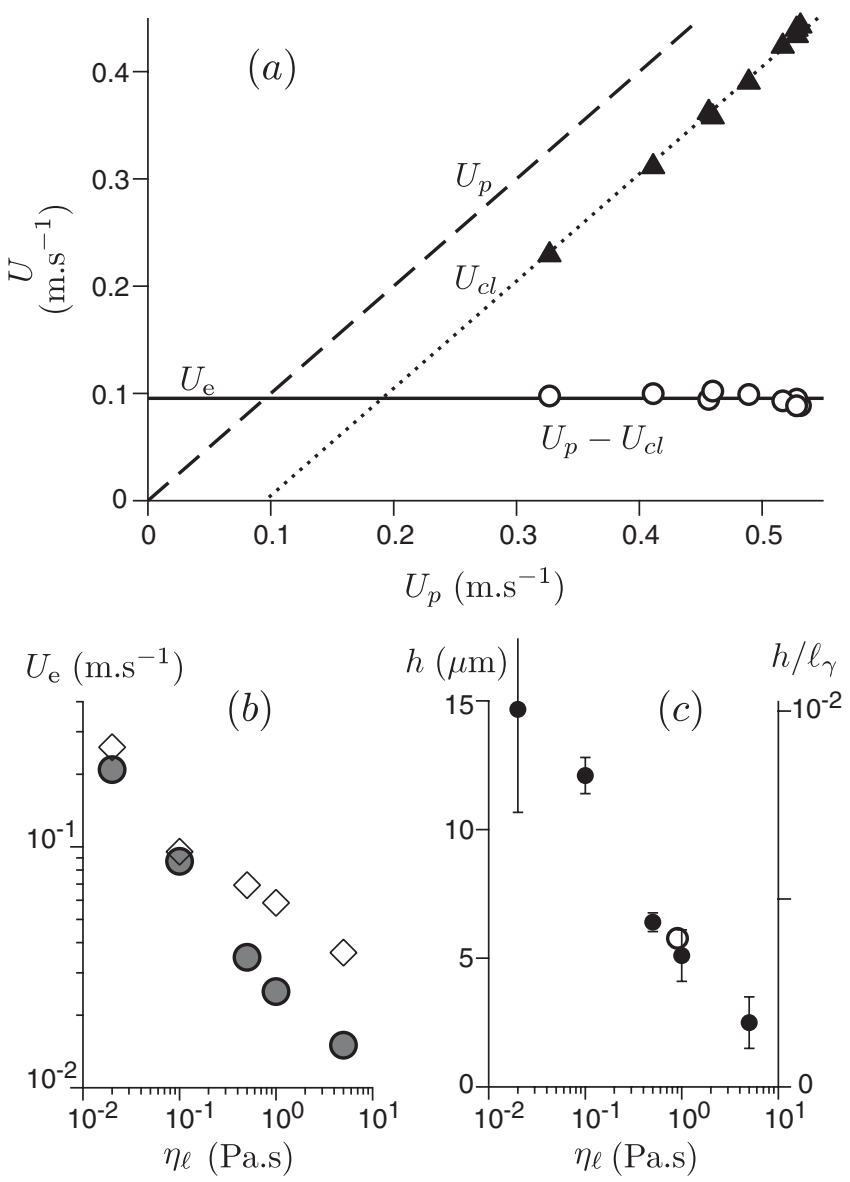

FIG. 2. (a) Contact line velocity $U_{\mathrm{cl}}$ (triangles) as a function of the plate velocity $U_{p}$, for $\eta_{\ell}=0.1 \mathrm{~Pa} \cdot \mathrm{s}$. The relative velocity $U_{p}-U_{\mathrm{cl}}$ (circles) is independent of the plate speed. (b) Entrainment speed $U_{e}$ for different liquid viscosities $\eta_{\ell}$, measured in two ways: the diamonds represent the relative velocity with respect to the plate, $U_{p}-U_{\mathrm{cl}}$, while the circles are the bridge rewetting speeds [see Fig. 1(d)]. (c) Film thickness $h$ for different $\eta_{\ell}$, taken at constant plate velocity $U_{p}=0.67 \mathrm{~m}$. $\mathrm{s}^{-1}$. The open circle was taken for a liquid jet of glycerol entraining air at the same speed [3]. The error bars are determined from a set of independent measurements.

Interesting dynamical structures are observed during air entrainment [Fig. 1(c)]. At the front of the film, the contact line develops a ridgelike shape that is common for dewetting of liquid films [24-26]. The peculiarity of the present experiment is that in this case the air is dewetted, not the liquid. An even more striking analogy with classical dewetting of liquids is the nucleation of nearly circular regions inside the film [Fig. 1(d)]. However, the circles now represent regions of rewetting, where the liquid reestablishes the contact with the solid [27]. These "rewetting bridges" can be considered as the inverse of the "dewetting holes," since the roles of air and liquid are exchanged. The bridges form nearly perfect circles, except for the two largest viscosities for which they are slightly stretched vertically; bridges that form after the plate is stopped are 
circular for all $\eta_{\ell}$. The radius of the bridge increases linearly with time, and the advancing contact lines collect the air inside a thick rim. While this is analogous to the inverse problem of the dewetting holes, the process is by no means symmetric: the rewetting circles grow with a velocity $U_{e}$ that is orders of magnitude faster than their dewetting counterparts, up to a factor 1000 for the liquids used in this study.

We further quantify the velocity of air entrainment for different liquid viscosities. A first measurement of $U_{e}$ is obtained from the growth velocities of the rewetting bridges as in Fig. 1(d). To ensure perfectly circular bridges, measurements are done immediately after stopping the plate. A second entrainment velocity is given by the plate velocity at which the film first appears. Since by definition, the contact line hardly moves downward at the transition, this velocity is most accurately determined from experiments well above the transition according to the following principle. By selecting a central part of the front of the air film, we first obtain the contact line velocity $U_{\mathrm{cl}}$ in the frame of the liquid reservoir. Figure 2(a) reports the measured values for $\eta_{\ell}=0.1 \mathrm{~Pa} \cdot \mathrm{s}$, showing that the contact line velocity increases linearly with plate velocity $U_{p}$. Interestingly, however, the relative velocity, $U_{e}=U_{p}-$ $U_{\mathrm{cl}}$, turns out to be independent of the plate velocity. Clearly, the film can only develop if the contact line can propagate downwards, i.e., when $U_{\mathrm{cl}}>0$ or $U_{p}>U_{e}$, thus providing an accurate determination of the critical speed. In analogy to receding contact lines this velocity appears to be an intrinsic property of the advancing contact line, independent of $U_{p}$ [21]. In other words, the structure of the contact line and the film appear to be completely independent from the bath: only the velocity relative to the plate matters. Indeed, the rewetting bridges display the same rimlike structure as the contact line front in Fig. 1(c). This suggests that we may consider the growth velocity of the rewetting bridges to be an independent measurement of the entrainment velocity. The resulting entrainment velocities $U_{e}$ are shown in Fig. 2(b), as a function of liquid viscosity $\eta_{\ell}$. Indeed, the two experimental definitions of $U_{e}$ agree very well for the smallest $\eta_{\ell}$ (diamonds are based on the front of the film, circles correspond to rewetting bridges). For larger $\eta_{\ell}$, the film rapidly destabilizes and it is more difficult to define the front of the film. This induces a difference between the two types of velocity measurements of about a factor 2; the bridge velocities are certainly more reproducible in this very viscous regime.

The key result of the velocity measurements is that, although $U_{e}$ decreases with liquid viscosity, the dependence is clearly much weaker than the expected $\sim 1 / \eta_{\ell}$. The entrainment speed is reduced by a factor 10 , while viscosity is varied by a factor 250 [Fig. 2(b)]. The data would be reasonably fitted by an exponent $-1 / 3$ or $-1 / 2$, but we refrain from claiming any definite power-law dependence. Since the liquid inertia is negligible for these highly viscous liquids, this means that the properties of the air must have a significant influence on the entrainment speed. On the other hand, the speed is not determined by the air alone, since that would yield no dependence on $\eta_{\ell}$ at all. To reveal the interplay between air and liquid phases, we introduce a dimensionless capillary number, $\mathrm{Ca}_{e}=$ $U_{e} \eta_{\ell} / \gamma$, that is based on the liquid viscosity. The experimental results are represented in Fig. 3, showing $\mathrm{Ca}_{e}$ versus the ratio of gas and liquid viscosities $\eta_{g} / \eta_{\ell}$ (circles and diamonds). Clearly, the capillary number for air entrainment displays a dependence that is much stronger than $\sim \ln \left(\eta_{\ell} / \eta_{g}\right)$, which is the scaling for air entrainment by liquid jets [2,3] and the prediction by Ref. [20]. The air thus has a much larger influence than expected. On the same figure, we collected data from the coating literature, based on tapes running continuously in a bath, showing a similar trend (various symbols, see caption). Note that in these experiments the contact line typically develops a sharp cusp from which small air bubbles are emitted, rather than an extended air film. At present there is no detailed understanding of the conditions necessary to trigger such cusps. In the case of receding contact lines, however, the instability of cusps are known to give similar values for $\mathrm{Ca}_{c}$ as straight contact lines $[21,28,29]$.

How can one understand the influence of the air, despite its very small viscosity? The key lies in the geometry of the

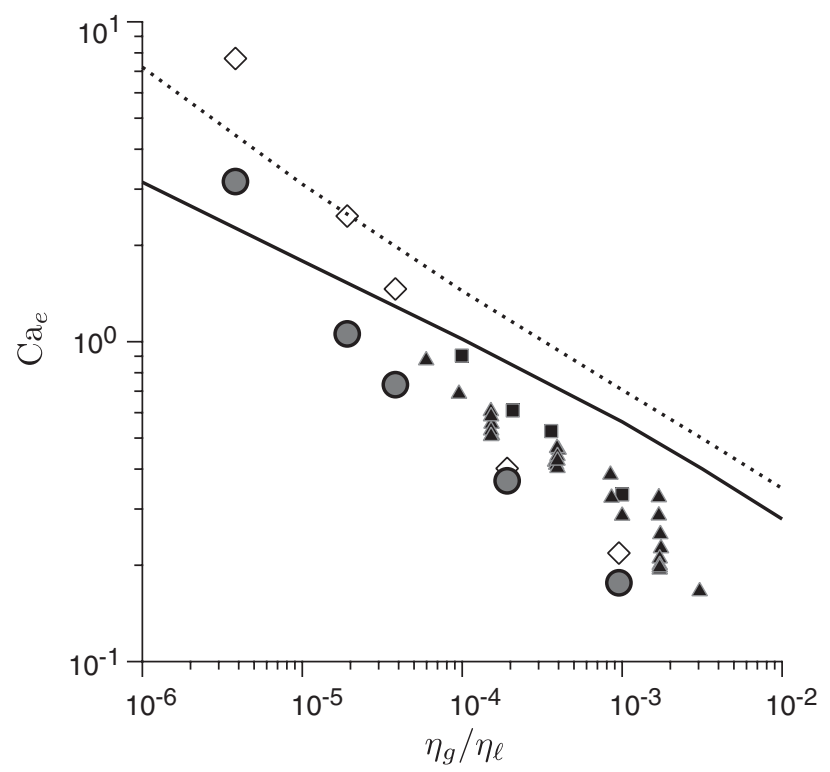

FIG. 3. Dimensionless entrainment speed, $\mathrm{Ca}_{e}=U_{e} \eta_{\ell} / \gamma$, versus the viscosity ratio $\eta_{g} / \eta_{\ell}$ for: silicon-oil/air $(\bullet, \diamond$, present data), silicon-oil/air ( $\boldsymbol{\square}$ after [18]) and various liquids/air ( $\boldsymbol{\Delta}$ after [36]). Curves: numerical results discussed in the Supplemental Material [23]. Corresponding parameters: oil slip lengths $\lambda_{\ell}=10^{-5} \ell_{\gamma}$, and air slip lengths $\lambda_{g}=10^{-4} \ell_{\gamma}$ (solid line) and $10^{-2} \ell_{\gamma}$ (dotted line), corresponding to mean free paths $\ell_{\mathrm{mfp}}=70 \mathrm{~nm}$ (solid) and $7 \mu \mathrm{m}$ (dotted). The theoretical curves do not suggest a definite exponent. 
flow near the entrainment transition. In the receding case, where the plate is pulled from the bath, it is well known that a liquid film appears whenever capillary forces can no longer balance the viscous drag. This transition occurs when the apparent, dynamic contact angle $\theta \rightarrow 0$ [30]. In this small contact angle limit, the rate of viscous dissipation scales as $\sim \eta_{\ell} U^{2} / h[14,22]$, where $h \simeq \theta x$ is the local thickness of the liquid, and $x$ the distance to the contact line. The $1 / h$ proportionality for the dissipation has two key consequences. First, the geometric confinement of liquid to a shallow wedge, as in Fig. 4(a), enhances viscous effects due to the factor $1 / \theta$. Second, the dependence $\sim 1 / x$ shows that dissipation is largest at small scales. As a consequence, the integrated viscous dissipation is due to all length scales, with comparable contributions from each decade between nanometer and millimeter scales. In the advancing case, however, the flow direction is reversed and the interface bends towards an angle $\theta \rightarrow \pi$. Figure 4(b) shows the classical solution for a perfect wedge by Huh and Scriven [31], illustrating that the flow of liquid becomes increasingly smooth when $\theta$ approaches $\pi$. In fact, it can be shown from these solutions that the liquid dissipation is $\sim(\pi-\theta)^{2}$ for angles close to $\pi$ [23]. While this would enable arbitrarily large speeds when $\theta \rightarrow \pi$, this mechanism is counteracted by the flow in the remaining wedge of air. The air becomes increasingly confined to an angle $\pi-\theta$, and the associated gas dissipation scales as $\sim 1 /(\pi-\theta)$. Hence, even the smallest gas viscosity ultimately gives a lower bound on the dissipation, and thus an upper bound on the advancing speed.

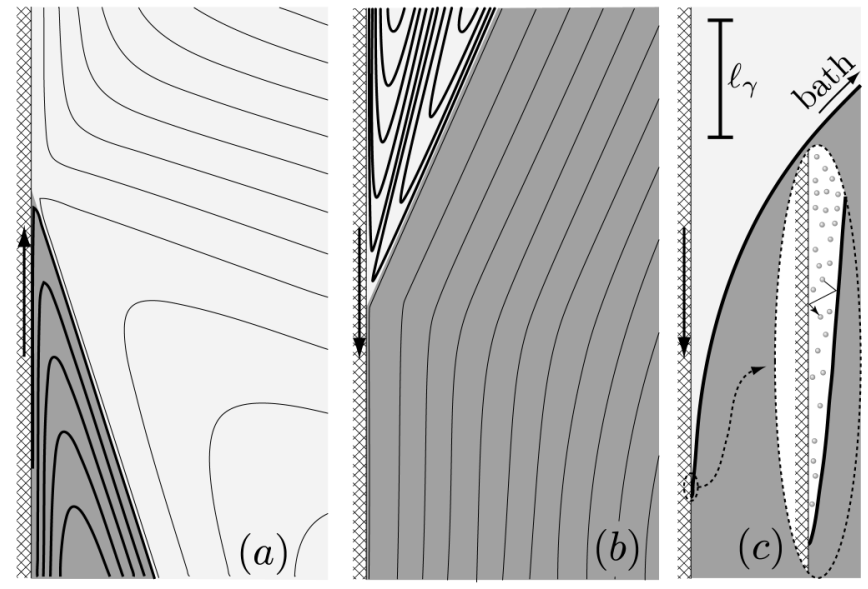

FIG. 4. Streamlines in a perfect wedge [31] of angle $\theta$ for (a) a receding contact line (with $\theta$ close to 0 ), and for (b) an advancing contact line (with $\theta$ close to $\pi$ ). In the advancing case, the viscous dissipation in the gas phase will dominate over the liquid phase due to the strongly confined circulation in the gas wedge. (c) Shape of the curved interface near the entrainment threshold. In a range of thickness $h$ between the molecular scale and the scale $\ell_{\gamma}$ of the bath, the local angle $\theta$ is close to $\pi$ so that dissipation is dominated by air. Inset: When the mean free path $\ell_{\mathrm{mfp}}$ is comparable to $h$, then air behaves as a Knudsen gas.
A quantitative theory for air entrainment requires a more detailed description of the interface shape, which in reality is strongly curved [Fig. 4(c)]. This means that the local angle $\theta$ should be considered to vary with $x$, and that Fig. 4(b) only provides a local estimate of the flow field. For air entrainment to occur, however, there must be a range of scales where the air flow is dominant, which from the above scaling arguments occurs when $(\pi-\theta)<$ $\left(\eta_{g} / \eta_{\ell}\right)^{1 / 3}$. Since each decade provides a comparable dissipative contribution, this qualitatively explains why total dissipation involves both $\eta_{\ell}$ and $\eta_{g}$. We further model this in the spirit of [32,33], extending the common lubrication approximation to large slopes and 2 phase flow-see Supplemental Material for details [23]. Numerical solution of the model provides the shape of the interface, as well as the capillary number for entrainment. The latter is shown in Fig. 3 (solid line). Since the model is derived by assuming small interface curvatures [32], it cannot be expected to be fully quantitative. Yet, it does capture the order of magnitude for $\mathrm{Ca}_{e}$ as well as the dependence on $\eta_{g} / \eta_{\ell}$.

In conclusion, we experimentally showed that the entrainment speed of advancing contact lines does not scale as $\gamma / \eta_{\ell}$, but exhibits a much weaker variation with liquid viscosity. We explain this by the influence of the air flow when the local angle of the interface is close to $\pi$. Can such a scenario explain the observed increase of entrainment speed when depressurizing the air $[18,19]$ ? A pressure reduction does not affect the dynamical viscosity of a gas [34]. However, as also mentioned in [18,19], it does increase the mean free path $\ell_{\mathrm{mfp}}$ by a factor $\ell_{\mathrm{mfp}} \sim p_{\mathrm{atm}} / p$. Since under atmospheric conditions $\ell_{\mathrm{mfp}} \approx 70 \mathrm{~nm}$, the mean free path is pushed well into the micron range when pressure is reduced by a factor 100 . The mean free path then becomes comparable to the film thickness measured experimentally. Since $\ell_{\mathrm{mfp}}$ sets the scale for the slip length $[23,35]$, we expect a substantial reduction of dissipation in the gas, and hence a larger entrainment velocity. Indeed, upon introducing $\ell_{\mathrm{mfp}}$ as the slip length, the model yields an increase of $\mathrm{Ca}_{e}$ (Fig. 3, dotted line). This provides the exciting perspective that depressurized air is a Knudsen gas when entrained by advancing contact lines [Fig. 4(c)].

We are grateful to J. Eggers and K. G. Winkels for valuable discussions. M. Fruchart is thanked for his help during preliminary experiments. T.S.C. acknowledges financial support by the FP7 Marie Curie Initial Training Network project ITN 215723.

[1] H. N. Oguz, A. Prosperetti, and A. Kolaini, J. Fluid Mech. 294, 181 (1995).

[2] J. Eggers, Phys. Rev. Lett. 86, 4290 (2001).

[3] E. Lorenceau, D. Quéré, and J. Eggers, Phys. Rev. Lett. 93, 254501 (2004).

[4] T. D. Blake and K. J. Ruschak, Nature (London) 282, 489 (1979). 
[5] J. de Jong, R. Jeurissen, H. Borel, M. van den Berg, H. Wijshoff, H. Reinten, M. Versluis, A. Prosperetti, and D. Lohse, Phys. Fluids 18, 121511 (2006).

[6] D. D. Joseph, J. Nelson, M. Renardy, and Y. Renardy, J. Fluid Mech. 223, 383 (1991).

[7] J.-T. Jeong and H. K. Moffatt, J. Fluid Mech. 241, 1 (1992).

[8] L. Xu, W. W. Zhang, and S. R. Nagel, Phys. Rev. Lett. 94, 184505 (2005).

[9] M. M. Driscoll, C. S. Stevens, and S. R. Nagel, Phys. Rev. E 82, 036302 (2010).

[10] P. Tsai, R.C. A. van der Veen, M. van de Raa, and D. Lohse, Langmuir 26, 16090 (2010).

[11] C. Josserand, P. Ray, and S. Zaleski, in 7th International Conference on Multiphase Flow ICMF 2010, Tampa, $F L, \quad 2010, \quad$ http://www.lmm.jussieu.fr/ zaleski/Papers/ ray-ICMF.pdf.

[12] S. Mandre, M. Mani, and M. P. Brenner, Phys. Rev. Lett. 102, 134502 (2009).

[13] M. Mani, S. Mandre, and M. Brenner, J. Fluid Mech. 647, 163 (2010).

[14] C. Duez, C. Ybert, C. Clanet, and L. Bocquet, Nature Phys. 3, 180 (2007).

[15] R. Ledesma-Aguilar, R. Nistal, A. Hernández-Machado, and I. Pagonabarraga, Nature Mater. 10, 367 (2011).

[16] R. Burley and B.S. Kennedy, Chem. Eng. Sci. 31, 901 (1976).

[17] P. G. Simpkins and V. J. Kuck, Nature (London) 403, 641 (2000).

[18] H. Benkreira and M. I. Khan, Chem. Eng. Sci. 63, 448 (2008).
[19] H. Benkreira and J. B. Ikin, Chem. Eng. Sci. 65, 1790 (2010).

[20] R. G. Cox, J. Fluid Mech. 168, 169 (1986).

[21] J. H. Snoeijer, G. Delon, M. Fermigier, and B. Andreotti, Phys. Rev. Lett. 96, 174504 (2006).

[22] D. Bonn, J. Eggers, J. Indekeu, J. Meunier, and E. Rolley, Rev. Mod. Phys. 81, 739 (2009).

[23] See Supplemental Material at http://link.aps.org/ supplemental/10.1103/PhysRevLett.108.204501 for the derivation of the two-phase lubrication equation and the experimental details.

[24] C. Redon, F. Brochard-Wyart, and F. Rondelez, Phys. Rev. Lett. 66, 715 (1991).

[25] J. C. Flitton and J. R. King, J. Eng. Math. 50, 241 (2004).

[26] J.H. Snoeijer and J. Eggers, Phys. Rev. E 82, 056314 (2010).

[27] G. Debregeas and F. Brochard-Wyart, J. Colloid Interface Sci. 190, 134 (1997).

[28] T. Podgorski, J. M. Flesselles, and L. Limat, Phys. Rev. Lett. 87, 036102 (2001).

[29] G. Delon, M. Fermigier, J. H. Snoeijer, and B. Andreotti, J. Fluid Mech. 604, 55 (2008).

[30] J. Eggers, Phys. Rev. Lett. 93, 094502 (2004).

[31] C. Huh and L. E. Scriven, J. Colloid Interface Sci. 35, 85 (1971).

[32] J. H. Snoeijer, Phys. Fluids 18, 021701 (2006).

[33] R. W. Hewson, J. Fluids Eng. 131, 041205 (2009).

[34] E. W. Lemmon and R. T. Jacobsen, Int. J. Thermophys. 25, 21 (2004).

[35] L. Bocquet, C. R. Acad. Sci. Paris, Série II 316, 7 (1993).

[36] R. Burley and B. S. Kennedy, Br. Polym. J. 8, 140 (1976). 